\title{
Heuristically Enhanced Feedback Control of Constrained Discrete-time Linear Systems*
}

\author{
M. SZNAIER $\dagger \ddagger$ and M. J. DAMBORG $\dagger$
}

\begin{abstract}
A theoretical framework to analyze the effects of using on-line optimization in the feedback loop is developed and applied to obtain a suboptimal controller guaranteed to yield asymptotically stable systems.
\end{abstract}

Key Words-Computer control; constrained systems; dynamic programming; feedback control; on-line operation; optimization; stability; suboptimal control; trees.

\begin{abstract}
Recent advances in computer technology have spurred new interest in the use of feedback controllers based upon on-line minimization for the control of constrained linear systems. Still the use of computers in the feedback loop has been hampered by the fact that the amount of time available for computation in most sampled data systems is not enough to achieve a complete solution using conventional algorithms. Several "ad hoc" techniques have been proposed, but their applicability is restricted by the lack of supporting theory. In this paper we present a theoretical framework to analyze the stability of the closed-loop system resulting from the use of on-line optimization in the feedback loop. Using these results we show that a suboptimal algorithm, based upon the use of heuristic search techniques, yields asymptotically stable systems, provided that enough computation power is available to solve at each sampling interval an optimization problem considerably simpler than the original. The controller presented in this paper is valuable for situations where the customary approaches of using Pontryagin's minimum principle or storing a family of extremal curves are not applicable due to limitations in the computational resources available.
\end{abstract}

\section{INTRODUCTION}

A LARGE CLASS of problems frequently encountered in practice involves the control of linear time-invariant systems with states and controls restricted to closed convex regions of the respective spaces. The origin of these constraints is diverse. They may represent physical limitations of the system (limitations of the materials, velocity of response of actuators, etc.) or they may originate in the process of modeling the physical system. The latter can be the case of a complex system represented by a piecewise linear model with coefficients obtained from

\footnotetext{
* Received 10 January 1989 ; revised 20 June 1989 received in final form 24 August 1989 . The original version of this paper was not presented at any IFAC meeting. This paper was recommended for publication in revised form by Associate Editor P. M. G. Ferreira under the direction of Editor H. Kwakernaak.

† Electrical Engineering Department, FT-10, University of Washington, Seattle, WA 98195, U.S.A.

$\ddagger$ Now with the Department of Electrical Engineering, Caltech, MS 116-81, Pasadena, CA 91125, U.S.A. Author to whom all correspondence should be addressed.
}

measurements performed at different operating points. In this case the states are constrained to remain in a neighborhood of the nominal operation points where the representation is sufficiently accurate. As examples of such systems we can mention advanced turbofan engines (Teren, 1977), hydroelectric reservoirs (Gutman, 1986) and robotic arms (Baker, 1987).

Control engineers have usually dealt with this class of problem by designing a controller ignoring the constraints and simply saturating the input (for a control constraint) or by switching on a controller that will attempt to steer the system from the boundary (when a state constraint is saturated). This approach, while appealing in its simplicity, is not easily applicable to higher order systems. Further, there is generally no guarantee that such an approach will yield an acceptable performance or even an asymptotically stable system. Another classical approach is to use dual mode controllers, where high gain feedback is used when the system is far from the constraints and low gain feedback is used when the system is approaching a constraint boundary. In using this approach, great care has to be exercised in order to avoid undesirable features such as limiting cycles.

There have been several recent attempts to design linear and nonlinear controllers for constrained systems. Van Til and Schmittendorf (1986) considered the controllability of discrete time systems with controls constrained to a convex set $\Omega$, but they proposed an off-line numerical algorithm that yields open-loop stabilizing control laws.

Vassilaki et al. (1988) solved the problem of stabilizing a linear discrete-time system under state and control constraints by using constant linear state feedback. Their controller is 
particularly attractive in its simplicity. However. it is clear that only a fraction of the feasible constrained problems admits a constant linear feedback solution. Hence the domain of application of Vassilaki's controller is limited to cases where enough control authority is available to allow for a stabilizing solution that does not require saturation of the control input. Moreover, optimal performance usually requires the control vector to be on a constraint boundary most of the time and this evidently requires a nonlinear controller capable of saturating.

Gutman and Hagander (1985) used quadratic Lyapunov functions to determine nonlinear feedback controllers for constrained systems. Their design procedure starts by finding a stabilizing linear feedback control law that does not violate the control constraints. Then, a quadratic Lyapunov function is constructed for the closed-loop system and a second control law is computed based upon this Lyapunov function. Finally, the two control laws are added and saturated. The use of a controller capable of saturating generally yields better performance than in the previous case. However, the applicability of this controller, as is the case with Vassilaki's controller, is limited by the requirement that a stabilizing constant linear feedback that does not violate the control constraints must exist. The use of quadratic Lyapunov functions further limits the domain of application since it is clear that in general non-quadratic functions must be used to determine the largest possible domain of attraction of the origin that is compatible with the constraints. Another serious drawback of the method is that it does not provide a systematic approach to the design. On the contrary, several steps of the design procedure involve an extensive trial and error process without guarantee of success [example 5.3 in Gutman and Hagander (1985)] and several parameters of the design must be tuned using simulations.

Alternatively, the problem can be stated as an optimization problem (Frankena and Sivan, 1979). Once the problem is cast in the form of an optimization problem, mathematical and dynamic programming techniques can be used to find the solution. Zadeh and Whalen (1962) solved a minimum time problem using Linear Programming. Fegley et al. (1971) explored stochastic and deterministic control design using Linear and Quadratic Programming. Mayne and Polak (1987) solved the problem using a penalty function-based algorithm.

Naturally, optimization approaches are appealing because they appear to guarantee an acceptable system response. However, in most cases the control law generated is an open-loop control that has to be recalculated entirely, with considerable computational effort, if the system is disturbed. Conceivably, the set of open-loop control laws could be used to generate a closed-loop control law by computing and storing a complicated field of extremals. However, this alternative is widely regarded as both complicated and expensive due to the extensive off-line computations and the amount of storage required. As a consequence, in most applications the optimal control law is not implemented.

To overcome this difficulty, Dreyfus (1964) introduced the concept of Open-Loop Optimal Feedback (OLOF). Given a discrete time system, the interval between samples is utilized to compute the optimal control law that will transfer the system from the present state to the desired final state. When this concept was introduced, its applicability was severely limited by the computing technology available at that time. However, recent advances in computing technology have reduced significantly the time required to compute the control law, spurring a new interest in controllers based upon on-line optimization. Recently, the OLOF concept has been applied successfully by Gutman (1986) to develop a Linear Programming-based regulator for a reservoir with a sampling time of $5 \mathrm{~min}$.

However, in most sampled control systems, the amount of time available between samples (typically ranging from tens of milliseconds up to a few seconds) may not be enough to compute a full solution to the problem, even allowing for advances in the technology in the foreseeable future. All previous implementations of the OLOF idea have dealt with this problem by simply using the last partial control law computed when they run out of time. As a result, we have the fundamental questions of whether the resulting closed-loop system is stable and whether the partial solution is a "sensible" control strategy, i.e. one that will steer the system in a convenient direction. To the best of our knowledge these questions have not been addressed and no theory exists that will allow an analysis of the resulting closed-loop system. The lack of a theory supporting the use of this ad hoc strategy limits the application of this class of controllers to cases where it is expected that, most of the time, there will be enough time available to compute a complete solution.

In this paper we will present a theoretical framework to analyze the stability properties of the closed-loop system resulting from the use of on-line optimization in the feedback loop. In the 
first portion of the paper we will present theoretical results guaranteeing the asymptotic stability of the system resulting from the use of a general control algorithm based upon an on-line minimization. In the second portion we will address the effects of the quantization of the control space on the controllability and stability of the system and we will specialize the results of Part 1 for the case of a suboptimal controller based upon heuristic search techniques. This algorithm has the advantage of providing an easy way of incorporating knowledge available about the solution into the controller, thus presenting the potential for a significant reduction of the computation time. Another advantage is that the resulting controller is easily generalizable to different performance indexes. Finally, examples of application of the proposed controller for minimum-time and quadratic cost cases will be presented.

\section{STATEMENT OF THE PROBLEM}

We will consider linear, time-invariant, controllable discrete time systems modeled by the difference equation:

$$
\mathbf{x}_{k+1}=A \mathbf{x}_{k}+B \mathbf{u}_{k}, \quad k=0,1, \ldots,
$$

with initial condition $\mathbf{x}_{0}$, and the constraints

$$
\mathbf{u}_{k} \in \Omega \subset R^{m}, \mathbf{x}_{k} \in \mathscr{G} \subseteq R^{n}
$$

where $\Omega$ and $\mathscr{G}$ are convex regions containing the origin in their interior, $\Omega$ is compact and where $\mathbf{x}$ indicates $x$ is a vector quantity. An additional hypothesis on the region $\mathscr{G}$, a constraint qualification hypothesis, will be introduced in a later section when the stability of the closed-loop system is analyzed. The objective is to find a sequence of admissible controls, $\mathbf{u}_{k}\left[\mathbf{x}_{k}\right]$, that minimizes a performance index of the form:

$$
J(\mathbf{x}, \mathbf{u}, N)=\sum_{k=0}^{N} L_{k}\left(\mathbf{x}_{k}, \mathbf{u}_{k}\right)
$$

with $L_{k}(\mathbf{x}, \mathbf{u}) \geq 0$ for all $\mathbf{x}$, $\mathbf{u}$ and $L_{k}(0,0)=0$. The notation $\mathbf{u}_{k}\left[\mathbf{x}_{k}\right]$ emphasizes the fact that a closed-loop solution is desired. We will call such a sequence a "global optimum". This problem will be denoted as problem $(P)$. Throughout this paper we will assume that $(P)$ is feasible for any initial condition in $\mathscr{G}$. (In Section 3 we will show how this assumption can be checked.) Note that the performance index (3) includes as particular cases minimum-time problems [when $L_{k}=1$ for all $\mathbf{x}, \mathbf{u} \neq 0, L_{k}(0,0)=0$ and $N$ is unspecified] and Linear Quadratic Regulators [when $N \rightarrow \infty$ and $\left.L_{k}=0.5 *\left(\mathbf{x}^{T} Q \mathbf{x}+\mathbf{u}^{T} R \mathbf{u}\right)\right]$.

In Sznaier and Damborg $(1987,1989)$ we proposed to solve this problem by using a feedback controller based upon the solution at each sampling interval of a sequence of suitable receding horizon type optimization problems, and we argued the soundness of the approach based on experimental results. In this paper we will present theoretical results showing that a similar algorithm yields an asymptotically stable system even in the face of computing time restrictions.

\section{DEFINITIONS AND THEORETICAL RESULTS}

In order to analyze the proposed controller we need to introduce the following definitions and theoretical results. We begin by formalizing the concept of feedback control:

Definition 1. A feedback control algorithm is a mapping $M: \mathscr{G} \times R \rightarrow \Omega$ defined by a finite computational procedure that yields a control law in terms of the present state of the system, i.e. $\mathbf{u}_{k}=M\left(\mathbf{x}_{k}, k\right)$.

The following definitions deal with the controllability aspects of the problem and in particular, with the effect of a quantization of the control space. These definitions will become particularly useful in the second part of the paper when a particular algorithm based upon a partition of the control space is analyzed. However, they are also useful outside this context, for instance to analyze the effect of using a computer with a finite word-length in the feedback loop.

Definition 2. The Null Controllable domain of (1) is the set of all points $\mathbf{x} \in \mathscr{G} \subseteq R^{n}$ that can be steered to the origin by applying a sequence of admissible controls $\mathbf{u}_{k} \in \Omega \subset R^{m}$, such that $\mathbf{x}_{k} \in \mathscr{G}, k=0,1, \ldots$ The Null Controllable domain of (1) will be denoted as $C_{x}$. The Null Controllable domain in $j$ or fewer steps will be denoted as $C_{j} \subseteq C_{\infty}$.

Definition 3. A (uniform) quantization $\Omega_{s}$ of a given set $\Omega \subseteq R^{m}$ is the set: $\Omega_{s}=\left\{\mathbf{u} \in \Omega: u_{i}=\right.$ $\left.n_{i} / s\right\}$, where $u_{i}$ is the $i$ th coordinate of $\mathbf{u}, n_{i}$ is integer and $s$ is a scaling factor. The quantity $1 / s$ will be called the norm of the quantization.

Definition 4. The system (1) is Quantized Null Controllable in a region $C \subseteq \mathscr{G}$ if, for any open set $S \subseteq \mathscr{G}$ containing the origin in its interior, there exist a number $s_{0}(C, S) \in R$ such that for all the quantizations $\Omega_{s}$ of $\Omega$ with $s \geq s_{0}$, there exists a sequence of admissible quantized controls $\mathbf{u}_{k} \in \Omega_{s}$ such that the system can be 
steered from any initial condition $\mathbf{x}_{1} \in C$ to $S$ without violating the state constraints.

In the next definition we formalize the concept of underestimate of the cost-to-go and we introduce a set where the constraints are not binding. These concepts will be used in Theorem 1 where we show that a particular sequence of approximations, based upon the use of underestimates, converges to the true cost-to-go.

Definition 5. Let $O$ be a convex open set containing the origin in its interior and such that, for all the optimal trajectories starting out in $O$, the constraints (2) are not effective and let $J_{0}(\mathbf{x})$ be the optimal cost-to-go from the state $\mathbf{x}$. A function $g: R^{n} \rightarrow R$ such that:

(1) $0 \leq g(\mathbf{x}) \leq J_{0}(\mathbf{x}) \quad \forall \mathbf{x} \in \mathscr{G}$

(2) $g(\mathbf{x})=J_{0}(\mathbf{x}) \quad \forall \mathbf{x} \in O$

will be called an underestimate of the cost-to-go, relative to the set $O$.

Note that from the definition it follows that once an optimal trajectory reaches the set $O$, it never leaves it. Several choices for $O$ and $g($. will be discussed later in the paper.

The following theorems present the basic theoretical foundations for the proposed algorithms.

Theorem 1. Let $O$ be the set introduced in Definition 5 and let $\mathbf{x}_{k}^{u}(\xi)$ be the (unconstrained) optimal trajectory corresponding to the initial condition $\xi \in O$. Finally let $g(\mathbf{x}): R^{n} \rightarrow R$ be an underestimate relative to $O$. Consider the following optimization problems:

$$
\begin{gathered}
\min _{u}\left\{J(\mathbf{x})=\sum_{k=0}^{N} L_{k}\left(\mathbf{x}_{k}, \mathbf{u}_{k}\right)\right\} \\
\min _{u}\left\{J_{m}(\mathbf{x})=\sum_{k=0}^{m-1} L_{k}\left(\mathbf{x}_{k}, \mathbf{u}_{k}\right)+g\left(\mathbf{x}_{m}\right)\right\}, \quad m<N
\end{gathered}
$$

subject to (1), with $\mathbf{u}_{k} \in \Omega \subset R^{m}$ and $\mathbf{x}_{k} \in \mathscr{G} \subseteq R^{n}$ and where $u=\left\{\mathbf{u}_{0}, \mathbf{u}_{1}, \ldots\right\}$. Then, an optimal trajectory, $\mathbf{x}_{k}^{0}, k=1,2, \ldots, m$ which solves (5), extended by defining $\mathbf{x}_{k}^{0}=\mathbf{x}_{k}^{u}\left(\mathbf{x}_{m}^{0}\right), \quad k=m+$ $1 \cdots N$, is also a solution of (4) provided that $\mathbf{x}_{m}^{0} \in O$.

The proof of this theorem follows from Bellman's dynamic programming theory.

From Theorem 1 it follows that the solution to the optimization problem (4) can be found by solving a sequence of optimization problems of the form (5) until a number $m$ and a trajectory $\mathbf{x}_{(\text {) }}$ such that $\mathbf{x}_{m} \in O$ are obtained. This concept is exploited in the following algorithm:

\section{Model algorithm (Algorithm $M$ )}

Begin:

(1) Let $\mathbf{x}_{k}$ be the current state of the system, $k$ the current time instant and $T$ the sampling interval. Then:

(i) If $\mathbf{x}_{k} \in O$ the solution coincides with that of the unconstrained problem.

(ii) If $\mathbf{x}_{k} \neq O$, solve a sequence of optimization problems of the form (5) until a number $m$ such that $\mathbf{x}_{m} \in O$ is found. Use as next control law, the first element of the control sequence corresponding to this solution.

(iii) If there is no more computation time available for searching and the region $O$ has not been reached, use the minimum partial cost trajectory that has been found.

(2) Repeat step 1 until the origin is reached. End.

It should be remarked that the proposed controller is a feedback controller and therefore can respond to the present condition. We successfully employed this algorithm in Sznaier and Damborg $(1987,1989)$ to design optimization based suboptimal controllers for constrained systems. However, the asymptotic stability of the resulting closed loop systems cannot be guaranteed when there is not enough time to reach the region where the cost-to-go is known (namely the region $O$ ), except in cases where the system starts out sufficiently close to that region. In this paper, in order to guarantee asymptotic stability, we will need to impose additional structure on our problem. However, to keep our problem as general as possible, we would like to impose the least amount of structure necessary. At the same time, we would like the domain of attraction of the origin to be as close as possible to the largest domain of attraction compatible with the constraints. These conditions can be formulated in the form of a qualification condition on the type of constraints allowed in $(P)$. Hence, we will impose a restriction on the type of constraints allowed in the problem and we will modify Algorithm M suitably.

\section{Constant qualification hypothesis}

We will consider admissible state regions of the form:

$$
\mathscr{G}=\{\mathbf{x}: G(x) \leq \boldsymbol{\gamma}\}
$$

where $\gamma \in R^{p}, \gamma_{i}>0$ and $G: R^{n} \rightarrow R^{p}$ has the following properties:

$$
\begin{gathered}
G(\mathbf{x})_{i} \geq 0, \quad i=1 \cdots p \forall \mathbf{x} \\
G(\mathbf{x})=0 \Leftrightarrow \mathbf{x}=0 \\
G(\mathbf{x}+\mathbf{y})_{i} \leq G(\mathbf{x})_{i}+G(\mathbf{y})_{i}, \quad i=1 \cdots p \forall \mathbf{x}, \mathbf{y} \in \mathscr{G} \\
G(\lambda \mathbf{x})=\lambda G(\mathbf{x}), \quad 0 \leq \lambda \leq 1 .
\end{gathered}
$$


Define now:

$$
v(\mathbf{x})=\max _{1 \leq i \leq p}\left\{G(\mathbf{x})_{i} / \gamma_{i}\right\}
$$

It is easy to show that, because of the positive definiteness of $G, v($.$) is a norm in the set \mathscr{G}$. We will denote this norm as $\|\cdot\|_{v}$. Note that $v($.$) is a$ "normalized distance" that measures the proximity of the constraints to saturation. Clearly $\|\mathbf{x}\|_{v} \leq 1$ for all $\mathbf{x} \in \mathscr{G}$ and $\|\mathbf{x}\|_{v}=1$ for $\mathbf{x}$ in the boundary of $\mathscr{G}, \partial \mathscr{G}$.

We will consider systems such that:

$$
\min _{\mathbf{u} \in \mathbf{\Omega}}\left\{\|A \mathbf{x}+B \mathbf{u}\|_{v}\right\}<1 \forall \mathbf{x} \in \partial \mathscr{G} \text {. }
$$

Condition (9) implies that for any initial condition on the boundary of the admissible region there exists a control that brings the system to its interior. Since the problem was assumed to be feasible in $\mathscr{G}$, the only effect of the additional constraints is to rule out the possibility of the system staying on the boundary for consecutive sampling instants. This condition can be checked using a number of techniques, depending on the actual form of $\mathscr{G}$ and $\Omega$. For example, if the constraints are of the form:

$$
\begin{aligned}
G(\mathbf{x}) & =|G \mathbf{x}| \quad \text { with } G \in R^{p * n} \text { and } \operatorname{rank}(G)=n \\
\Omega & =\{\mathbf{u}: W \mathbf{u} \leq \rho\} \quad \text { with } W \in R^{q * m} \text { and } \rho \in R^{q}
\end{aligned}
$$

then they can be reduced to a system of linear inequalities and (9) can be checked using linear programming. In the case where:

$$
\begin{aligned}
G(\mathbf{x}) & =\left(\mathbf{x}^{T} Q \mathbf{x}\right)^{1 / 2} \\
\Omega & =\left\{\mathbf{u}:\left(\mathbf{u}^{\mathrm{T}} \mathbf{R} \mathbf{u}\right)^{1 / 2} \leq \rho\right\}, Q, R \text { positive definite }
\end{aligned}
$$

then a sufficient condition for the satisfaction of constraint (9) can be found based upon the singular value decomposition of the matrices $Q$ and $R$, using a technique similar to the one employed in Example 3, Section 6.

Note that (6), (7) and the convexity of $\Omega$ imply that the satisfaction of (9) guarantees the feasibility of the problem in $\mathscr{G}$.

Theorem 2. Consider problem $(P)$ with the additional constraints (6), (7) and (9). Then, there exists a control sequence $\mathscr{U}=\left\{\mathbf{u}_{0}, \ldots,\right\}$, $\mathbf{u}_{k} \in \Omega$ such that:

$$
\left\|\mathbf{x}_{k+1}\right\|_{v}<\left\|\mathbf{x}_{k}\right\|_{v}, k=0,1 \cdots \forall \mathbf{x}_{k} \in \mathscr{G}-O .
$$

Proof. From (9) it follows that:

$$
\min _{\mathbf{u} \in \mathbf{\Omega}}\|A \mathbf{x}+B \mathbf{u}\|_{v}<\|\mathbf{x}\|_{v}=1 \forall \mathbf{x} \in \partial \mathscr{G}
$$

Since the origin is an interior point of $\mathscr{G}$ by hypothesis, it follows from the convexity of $\mathscr{G}$ that for any $\mathbf{x} \in$ int $\{\mathscr{G}\}$, there exist $\lambda, 0 \leq \lambda \leq 1$, and $\mathbf{y} \in \partial \mathscr{G}$ such that $\mathbf{x}=\lambda \mathbf{y}$. Hence from (7),
(9), and the convexity of $\Omega$ we have:

$$
\begin{gathered}
\min _{\mathbf{u} \in \Omega}\left\{\|A \mathbf{x}+B \mathbf{u}\|_{v}\right\}=\min _{\mathbf{u} \in \mathbf{\Omega}}\left\{\|\lambda A \mathbf{y}+B \mathbf{u}\|_{v}\right\} \\
\leq \min _{\mathbf{u} \in \Omega}\left\{\|\lambda A \mathbf{y}+\lambda B \mathbf{u}\|_{v}\right\} \\
=\lambda \min _{\mathbf{u} \in \Omega}\left\{\|A \mathbf{y}+B \mathbf{u}\|_{v}\right\}<\lambda\|\mathbf{y}\|_{v}=\|\mathbf{x}\|_{v} .
\end{gathered}
$$

Therefore u can be selected such that (10) is satisfied for all points in $\mathscr{G}$. $\diamond$.

Corollary 1. The problem $\left(\boldsymbol{P}^{\prime}\right)$ defined as problem $(P)$ with the additional constraints $(6)$, (7), (9) and (10) is feasible, i.e. there exists at least one control sequence such all the constraints are satisfied.

From Theorem 2 and its corollary, it follows that the application of Algorithm $M$ to problem $\left(P^{\prime}\right)$ yields a control law such that the v-norm of the state is monotonically decreasing in $\mathscr{G}-O$. In the next theorem we use this result to show the asymptotic stability of the closed-loop system.

Theorem 3. The closed-loop system resulting from the application of Algorithm $\mathrm{M}$ to problem $\left(P^{\prime}\right)$ is asymptotically stable.

Proof. It is clear that $\mathbf{x}_{m}$ is always bounded for all $m$ since $\mathbf{x}_{m} \in \mathscr{G}$. Hence we only have to show that $\mathbf{x}_{m} \rightarrow 0$.

(a) Consider first the case when $\mathbf{x}_{0} \in O$. By definition, the constraints are not binding for all the optimal trajectories starting out in $O$ and the optimal cost-to-go is known and equal to $g(\mathbf{x})$. It follows that $g(\mathbf{x})$ is a Lyapunov function for the system and hence it is asymptotically stable in $O$.

(b) Consider now the case where $\mathbf{x}_{0} \in \mathscr{G}-O$. We will show that there exists $m_{0}$ such that $\mathbf{x}_{m_{0}} \in O$. Define:

$$
\begin{aligned}
& \mu=\min _{\mathbf{x} \in \partial O}\|\mathbf{x}\|_{v} \\
& \delta=\max _{\mathbf{x} \in \mathscr{S}_{\mathcal{G}}-O}\left\{\left\|\mathbf{x}_{k+1}\right\|_{v}-\left\|\mathbf{x}_{k}\right\|_{v}\right\}
\end{aligned}
$$

where $\mathbf{x}_{k+1}=A \mathbf{x}_{k}+B M\left(\mathbf{x}_{k}\right)$.

Clearly $\mu>0$ and if $\|\mathbf{y}\|_{v}<\mu$ then $\mathbf{y} \in O$. From the definition of $\left(P^{\prime}\right)$ and (10) it follows that $\delta<0$. Hence:

$$
\begin{gathered}
\left\|\mathbf{x}_{1}\right\|_{v}<\left\|\mathbf{x}_{0}\right\|_{v}+\delta \\
\vdots \\
\left\|\mathbf{x}_{n}\right\|_{v}<\left\|\mathbf{x}_{0}\right\|_{v}+n \delta
\end{gathered}
$$

and there exist $n_{0}$ such that $\mathbf{x}_{n_{0}} \in O$. Once the system reaches the region $O$, part (a) of the proof applies and $\mathbf{x}_{m} \rightarrow 0$. This completes the proof. 
Theorems 1 through 3 show that Algorithm M applied to the modified problem $\left(P^{\prime}\right)$ yields an asymptotically stable system. In applying this algorithm, we make the implicit assumption that there is enough computation time available to allow for the computation of a control law $\mathbf{u}=M(\mathbf{x})$ such that all the constraints are satisfied. Specifically, we assume that there is at least enough time to solve a problem of the form (5) with $m=1$.

Note that Algorithm $M$ is a "conceptual" algorithm, since it cannot be implemented until a finite procedure to perform the optimization required by step 1 (ii) is specified. In the next section we will present a specific algorithm to perform the on-line minimization.

\section{CONTROL ALGORITHM}

In Sznaier and Damborg (1987) we presented a suboptimal feedback controller for a class of systems based upon the use of Quadratic Programming for on-line minimization. In this section we investigate the application of Heuristically Enhanced Optimal Control as an alternative for on-line minimization. In this approach, suggested by Guez (1986), the problem is approximately solved by partitioning the control space $\Omega$ into a finite set $\Omega_{s}$ as shown in Fig. 1. The attainable domain from the initial condition, using controls in $\Omega_{s}$, can be represented now as a tree with each node corresponding to one of the attainable states. Hence the original optimal control problem is recast as a tree search, with the approximation resulting from the control quantization. The resulting tree can be scanned efficiently for minimum cost paths using heuristic search techniques, based upon an underestimate of the cost-to-go (Winston, 1984). In order to analyze the stability properties of this controller we will address the effect of the quantization of the control space, making use of the definitions introduced in the previous sections.

The following results show that if $(P)$ is feasible, then $\left(P^{\prime}\right)$ is also feasible, even when the controls are restricted to a quantization $\Omega_{s}$ of $\Omega$. It follows then that conditions (6), (7) and (9) are sufficient conditions to guarantee quantized null controllability.

In the following lemma we introduce a quantity $(\Lambda)$ that gives a measure of how much the set $O$ can be "magnified" before exceeding
$\Omega$
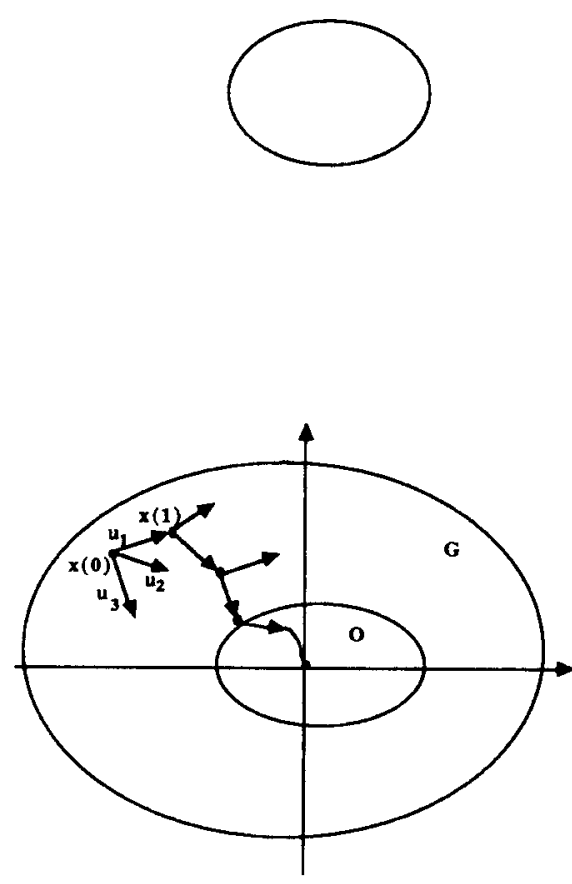

$\Omega$

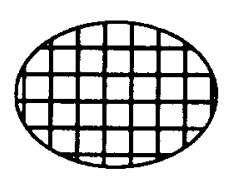

桨

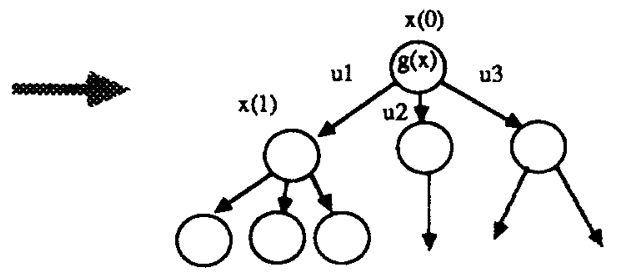

Estimated cost to go for the trajectory $x(0)-x(n)$ :

$$
\begin{aligned}
& J_{n}(x, u)=\sum_{k=0}^{n-1} L(x(\underbrace{k}), u(k))+g(\underbrace{x(n)}) \\
& \text { actual cost heuristic } \\
& \text { approximation }
\end{aligned}
$$

FIG. 1. Diagram of Algorithm H (heuristically enhanced optimal control). 
the constraints. Then, we use this quantity to get a lower bound on the maximum amount that the norm of the present state of the system $\left(\|\mathbf{x}\|_{v}\right)$ can be decreased in one stage.

Lemma 1. Consider the region $\mathscr{G}-O$. Let:

$$
\Lambda=\min _{\mathbf{x} \in \partial O}\left\{\lambda:\left(\frac{1}{\lambda} \mathbf{x}\right) \in \partial \mathscr{G}\right\} .
$$

Then:

$$
\begin{aligned}
\min _{\mathbf{x} \in \mathcal{G}-O}\left\{\|\mathbf{x}\|_{v}-\min _{\mathbf{u} \in \Omega}\|A \mathbf{x}+B \mathbf{u}\|_{v}\right\} \\
>\Lambda \min _{\mathbf{y} \in \partial \mathscr{S}}\left\{1-\min _{\mathbf{u} \in \Omega}\|A \mathbf{y}+B \mathbf{u}\|_{v}\right\}
\end{aligned}
$$

Proof. Given any $\mathbf{x} \in \mathscr{G}-O$ it can be expressed as $\lambda_{0} \mathbf{y}_{0}$ with $\mathbf{y}_{0} \in \partial \mathscr{G}$ and $0<\lambda_{0} \leq 1$. Then:

$$
\begin{aligned}
& \|\mathbf{x}\|_{v}-\min _{\mathbf{u} \in \mathbf{\Omega}}\|A \mathbf{x}+B \mathbf{u}\|_{v} \\
& =\left\|\lambda_{0} \mathbf{y}_{0}\right\|_{v}-\min _{\mathbf{u} \in \boldsymbol{\Omega}}\left\|A \lambda_{0} \mathbf{y}_{0}+B \mathbf{u}\right\|_{v} \\
& \geq\left\|\lambda_{0} \mathbf{y}_{0}\right\|_{v}-\min _{\mathbf{u} \in \mathbf{\Omega}}\left\|A \lambda_{0} \mathbf{y}_{0}+B \lambda_{0} \mathbf{u}\right\|_{v} \\
& =\lambda_{0}\left\{\left\|\mathbf{y}_{0}\right\|_{v}-\min _{\mathbf{u} \in \Omega}\left\|A \mathbf{y}_{0}+B \mathbf{u}\right\|_{v}\right\} \\
& \geq \min _{\substack{\mathbf{y} \in \mathcal{Z}^{\mathcal{S}} \\
\lambda \mathbf{y} \in G-O}} \lambda\left\{\|\mathbf{y}\|_{v}-\min _{\mathbf{u} \in \mathbf{\Omega}}\|A \mathbf{y}+B \mathbf{u}\|_{v}\right\} \\
& \geq \min _{\substack{\mathbf{y} \in \partial \mathscr{g} \\
\lambda \mathbf{y} \in \partial O}} \lambda\left\{\|\mathbf{y}\|_{v}-\min _{\mathbf{u} \in \Omega}\|A \mathbf{y}+B \mathbf{u}\|_{v}\right\} \\
& \geq \Lambda \min _{\mathbf{y} \in \partial \mathfrak{G}}\left\{1-\min _{\mathbf{u} \in \boldsymbol{\Omega}}\|A \mathbf{y}+B \mathbf{u}\|_{v}\right\}
\end{aligned}
$$

since $\|\mathbf{y}\|_{v}=1$ for $\mathbf{y} \in \partial \mathscr{G}$

Note that in this theorem we consider a "worst-case" type situation by essentially considering a ray from the origin to the boundary of the constraint set, $\partial \mathscr{G}$, and then, in the last inequality, decoupling the scale factor $(\Lambda)$ from the orientation. Note also that $\Lambda$ is strictly positive.

In the next theorem we address the feasibility of $\left(P^{\prime}\right)$ when the controls are restricted to a quantization $\boldsymbol{\Omega}_{s}$.

Theorem 4. Assume that $G$ satisfies the conditions (7). Then, there exists a number $s_{0}$ such that for all quantizations $\Omega_{s}$ of $\Omega$ with $s \geq s_{0}$, the constraint (10) can be satisfied for all $\mathbf{x}_{k} \in \mathscr{G}-O$ and $\mathbf{u}$ restricted to $\Omega_{s}$.

Proof. Consider a quantization $\Omega_{s}$ of $\Omega$ with norm $\frac{1}{s}$ and a point $\mathbf{x} \in \mathscr{G}-O$. Let $\mathbf{t}_{0}$ be an element of $\Omega_{s}$ where $\|A \mathbf{x}+B \mathbf{u}\|_{v}$ achieves its minimum over all elements in $\Omega_{s}$ (note that this minimization is well defined since $\Omega_{s}$ is a finite set), i.e.

$$
\mathbf{t}_{0}=\underset{\mathbf{u} \in \Omega_{s}}{\operatorname{argmin}}\|A \mathbf{x}+B \mathbf{u}\|_{v} .
$$

Then, there exist a $\delta \mathbf{u}, \delta u_{i} \leq 1 / s$ such that:

$$
\left\|A \mathbf{x}+B \mathbf{t}_{0}\right\|_{v}=\min _{\mathbf{u} \in \Omega}\|A \mathbf{x}+B \mathbf{u}+B \delta \mathbf{u}\|_{v} .
$$

From (7) and (8) we have that:

$$
\begin{array}{r}
\min _{\mathbf{t} \in \Omega_{s}}\|A \mathbf{x}+B \mathbf{t}\|_{v}=\min _{\mathbf{u} \in \Omega}\|A \mathbf{x}+B \mathbf{u}+B \boldsymbol{\delta} \mathbf{u}\|_{v} \\
\leq \min _{\mathbf{u} \in \Omega}\|A \mathbf{x}+B \mathbf{u}\|_{v}+\max _{1 \leq i \leq p}\left[\frac{G(B \boldsymbol{\delta} \mathbf{u})_{i}}{\gamma_{i}}\right] .
\end{array}
$$

From (7), it can be shown that $G(\boldsymbol{\delta x}) \rightarrow 0$ as $\delta \mathbf{x} \rightarrow 0$. Hence, it follows that $s$ may be chosen large enough so that:

$$
\max _{1 \leq i \leq p}\left[\frac{G(B \boldsymbol{\delta} \mathbf{u})_{i}}{\gamma_{i}}\right]<\Lambda \min _{\mathbf{y} \in \partial \mathscr{S}}\left\{1-\min _{\mathbf{u} \in \Omega}\|A \mathbf{y}+B \mathbf{u}\|_{v}\right\}
$$

since (9) guarantees that the right-hand side of (21) is strictly positive. From Lemma 1 we have:

$$
\begin{aligned}
\Lambda \min _{\mathbf{y} \in \partial \mathscr{S}}\left\{1-\min _{\mathbf{u} \in \mathbf{\Omega}}\|A \mathbf{y}+B \mathbf{u}\|_{v}\right\} \\
<\|\mathbf{x}\|_{v}-\min _{\mathbf{u} \in \Omega}\|A \mathbf{x}+B \mathbf{u}\|_{v} .
\end{aligned}
$$

Hence, from (20), (21) and (22) it follows that:

$$
\begin{aligned}
\min _{\mathbf{t} \in \Omega_{s}} \| A \mathbf{x}+ & B \mathbf{t}\left\|_{v} \leq \min _{\mathbf{u} \in \mathbf{\Omega}}\right\| A \mathbf{x}+B \mathbf{u} \|_{v} \\
& +\max _{1 \leq i \leq p}\left[\frac{G(B \delta \mathbf{u})_{i}}{\gamma_{i}}\right]<\|\mathbf{x}\|_{v} . \diamond
\end{aligned}
$$

Corollary 2. The system (1) is Quantized Null Controllable in $\mathscr{G}$. The proof follows from Theorem 4 by selecting a set $O$ such that $O \subseteq S$, where $S$ is an arbitrary open set containing the origin in its interior, as required by Definition 4 , Section 3.

From Theorem 4 it follows that $\left(P^{\prime}\right)$ can be solved by using quantized controls in the region $\mathscr{G}-O$ and switching to non-quantized controls inside $O$. This result is the basis of the following feedback control algorithm.

\section{Algorithm H (Heuristically Enhanced Control)} Begin:

(1) Determine the set $O$ and a function $g(\mathbf{x}): R^{n} \rightarrow R, \quad 0 \leq g(\mathbf{x}) \leq J_{0}(\mathbf{x})$ for all $\mathbf{x} \in \mathscr{G}$, $g(\mathbf{x})=J_{0}(\mathbf{x})$ for all $\mathbf{x} \in O$. The function $g(\mathbf{x})$ will be used as an heuristic guideline for estimating the cost-to-go in the search for 
optimal trajectories, hence it should be close to $J_{0}(\mathbf{x})$.

(2) Determine the scaling factor $s$ for the quantization of $\Omega$. A lower bound of $s$ can be determined from Theorem 4 , to assure that the set $O$ can be reached using the quantized controls. The upper bound of $s$ depends on the amount of time available for computation. There is a trade-off between computation time and the proximity of the quantized trajectory to the true minimum.

Note that steps 1 and 2 are performed during the controller design state, not during its use.

(3) Let $\mathbf{x}_{k}$ be the current state of the system, $k$ the current time instant and $T$ the sampling interval. Then:

(i) If $\mathbf{x}_{k} \in O$ the solution coincides with that of the unconstrained problem.

(ii) If $\mathbf{x}_{k} \notin O$, generate a tree by considering all the possible trajectories starting at $\mathbf{x}_{k}$, with controls $\mathbf{u}_{k} \in \Omega_{s}$. Nodes that do not verify the constraints (2) or (10) are not added to the tree. This process generates a finite tree since we are dealing with a finite set $\Omega_{s}$. Search the tree for a minimum cost trajectory to the origin using heuristic search algorithms. Note that once a trajectory reaches the region $O$, then the cost to proceed from the first point of the trajectory interior to $O, \mathbf{x}^{*}$, to the origin is given by $g\left(\mathbf{x}^{*}\right)$. Therefore, if the sampling time $T$ is long enough to allow the algorithm to expand the tree until $O$ is reached, the results of Theorem 1 imply that the solution found is the true optimal solution.

(iii) If there is no more computation time available for searching and the region $O$ has not been reached, use the minimum partial cost trajectory that has been found. Care should be exercised in order to assure that the trajectory is selected based upon the last completely explored level of the tree. The algorithm for generating and exploring the tree is listed in the Appendix.

(4) Repeat step 3 until the origin is reached. End.

Figure 1 shows a diagram of the algorithm. From Theorems 3 and 4 it follows that the resulting closed-loop system is asymptotically stable in $\mathscr{G}$, even when there is not enough time to completely expand the tree, provided that there is enough time to completely expand its first level. This provides a lower bound on the computational resources necessary to implement the algorithm successfully.
5. APPLICATION TO MINIMUM TIME PROBLEMS

In this section we investigate the application of the proposed controller to minimum time problems, i.e. the case when $L_{k}\left(\mathbf{x}_{k}, \mathbf{u}_{k}\right)=1 \forall \mathbf{x}_{k}$, $\mathbf{u}_{k} \neq 0 ; L_{k}(0,0)=0$ and $N$ is unspecified. It is clear that in this case a lower bound, $g(\mathbf{x})$, of the cost-to-go can be obtained by estimating the number of steps necessary to reach the origin, taking into account only the control constraints (in which case an approximation to the Null Controllability regions can be found based upon the geometric properties of the set $\Omega$ and the matrices $A$ and $B$ ). It is also clear that inside the set $O=C_{1}^{u} \cap \mathscr{G}$ the constraints (2) are not effective (where $C_{1}^{u}$ denotes the Null Controllability region in 1 step taking into account only the control constraints).

Let $B_{k} \subseteq R^{n}$ be a ball in state space such that $C_{k}^{u} \subseteq B_{k}$ and let $\boldsymbol{x}$ be the present state of the system. Then:

(i) if $\mathbf{x} \in O=C_{1}^{u} \cap \mathscr{G}$ use as the control law the solution to the unconstrained problem.

(ii) if $\mathbf{x} \notin O$ find the largest integer $m$ such that $\mathbf{x} \notin B_{m}$ and use $g(\mathbf{x})=m+1$ as a lower bound of the cost.

Note that the minimum time problem is potentially degenerate since there may exist more than one control that transfers the system from the region $C_{k+1}$ to the region $C_{k}$ hence yielding the same value of the performance index. To maintain a well ordered tree, an auxiliary cost function to remove degeneracies must be provided. For example this auxiliary cost could be the energy involved in the transition or the distance from the new state to the origin.

\section{Example 1}

Consider the spinning space station with a single axis of symmetry problem (Athans and Falb, 1966; Van Til and Schmittendorf, 1986). The station is controlled by means of a single jet placed on the body and allowed to rotate to any angle in a plane normal to the symmetry axis. Selecting as state variables the angular velocities around a pair of axis also perpendicular to the symmetry axis, the system can be represented by:

with:

$$
\mathbf{x}_{k+1}=A \mathbf{x}_{k}+B \mathbf{u}_{k}
$$

$$
\begin{gathered}
A=\left(\begin{array}{cc}
\cos T & \sin T \\
-\sin T & \cos T
\end{array}\right) \\
B=\left(\begin{array}{cc}
\sin T & (1-\cos T) \\
(\cos T-1) & \sin T
\end{array}\right) \\
\mathscr{G}=\left\{\mathbf{x} \in R^{2}:\|\mathbf{x}\|_{2} \leq R_{x}\right\} \\
\Omega=\left\{\mathbf{u} \in R^{2}:\|\mathbf{u}\|_{2} \leq 1\right\}
\end{gathered}
$$

where $T$ is the sampling interval. 


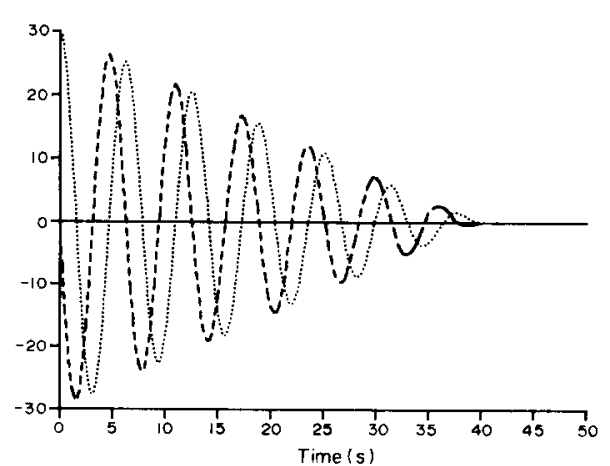

FIG. 2a. States for Example 1.

Note that in this case $A$ is an orthogonal matrix and that $B * B^{T}=\alpha^{2} I$ where $\alpha^{2}=2(1-$ $\cos T$ ). The one step controllability region $C_{1}^{u}$ is the circle given by:

$$
C_{1}^{u}=\left(-A^{-1} B\right) \Omega=\alpha \Omega .
$$

Since $C_{1}^{u}$ is rotationally invariant under $A$ it is easily shown that the $n$-step null controllability region is the circle given by:

$$
C_{n}^{u}=n C_{1}^{u}
$$

In this particular case, since the controllability regions are circles, the lower bound of the cost-to-go reduces to:

$$
n(\mathbf{x})=\left\lceil\left(\frac{\|\mathbf{x}\|_{2}}{\alpha}\right)\right\rceil
$$

To remove degeneracies, we employed the norm of the state as an auxiliary heuristic. Figure 2 shows the response of the controller for $R_{x}=30$, with initial condition $\mathbf{x}^{T}=(30,0)$, a sampling interval of $2.5 \mathrm{~s}$ and a quantization $\|\mathbf{u}\|_{\infty} \leq 0.04$ determined using Theorem 4. To simulate realistic conditions the algorithm was constrained to use a maximum computation time of $2.0 \mathrm{~s}$. The resulting control law coincides with the control found by Van Til and Schmittendorf (1986) using an off-line numerical algorithm.

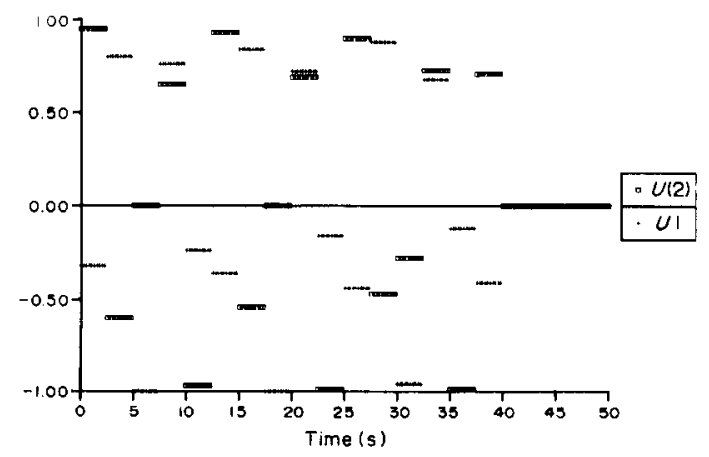

FIG. 2b. Controls for Example 1.

\section{APPLICATION TO LINEAR QUADRATIC} REGULATORS

In this section we investigate Linear Quadratic Regulators, i.e. the special case of problem $(P)$ where $N \rightarrow \infty$ and $L_{k}=\frac{1}{2}\left(\mathbf{x}_{k}^{T} Q \mathbf{x}_{k}+\mathbf{u}_{k}^{T} R \mathbf{u}_{k}\right)$. Let $K_{0}$ be the optimal feedback gain for the unconstrained system and $S$ the solution to the associated Algebraic Riccati Equation. Finally, let $X_{K_{0}} \subseteq \mathscr{G} \subseteq R^{n}$ be the region defined as:

$X_{K_{0}}=\{\mathbf{x} \in \mathscr{G}$ : for the optimal trajectory starting at $\mathbf{x}$ and using the feedback law $\mathbf{u}_{k}=-K_{0} \mathbf{x}_{k}$ then:

(1) $\mathbf{u}_{k} \in \Omega$ for every $k$,

(2) the states $\mathbf{x}_{k}$ of the closed-loop system never leave the region $X_{K_{0}}\left(\right.$ and hence $\left.x_{k} \in \mathscr{G} \forall k\right)$,

(3) $\left.\lim _{k \rightarrow \infty} \mathbf{x}_{k}=0\right\}$.

Theorems 3.1 and 3.2 in Sznaier and Damborg (1989) show that $X_{K_{0}}$ is non-empty and that a region $Y \subseteq X_{K_{0}}$ can be constructed by finding points contained in $X_{K_{0}}$ and joining them in a convex polyhedron.

It is clear that $g_{1}(\mathbf{x})=\frac{1}{2} \mathbf{x}^{T} S \mathbf{x}$ is a suitable lower bound of the cost-to-go, since it gives the optimal cost-to-go for the unconstrained problem. Therefore the algorithm can be applied using $g_{1}(\mathbf{x})$ as heuristic and $X_{K_{0}}$ as the set $O$.

\section{Example 2}

Consider the test system utilized in Sznaier and Damborg $(1987,1989)$ given by:

$$
\mathbf{x}_{k+1}=A \mathbf{x}_{k}+B \mathbf{u}_{k}
$$

with:

$$
\begin{aligned}
A & =\left(\begin{array}{ll}
1.0 & 0.2212 \\
0.0 & 0.7788
\end{array}\right) \quad B=\left(\begin{array}{l}
0.0288 \\
0.2212
\end{array}\right) \\
G & =\left(\begin{array}{ll}
1.0 & 0.0 \\
0.0 & 1.0 \\
1.5 & 1.0
\end{array}\right) \quad \gamma=\left(\begin{array}{l}
1.2 \\
0.3 \\
1.8
\end{array}\right) \\
\Omega & =\{u \in R:|u| \leq 0.5\} \\
\mathscr{G} & =\left\{\mathbf{x} \in R^{2}:|G \mathbf{x}| \leq \gamma\right\}
\end{aligned}
$$

and a sampling time of $0.25 \mathrm{~s}$. The objective is to drive the system to the origin with unspecified final time and with minimum energy, so the matrices $Q$ and $R$ are selected to be the identity of appropriate dimensions. The unconstrained LQ solution is given by:

$$
\mathbf{u}=-K_{0} \mathbf{x}, K_{0}=\left(\begin{array}{ll}
0.8831 & 0.8811
\end{array}\right)
$$

It is easily verified, by checking the vertices (Sznaier and Damborg, 1987), that the region:

$$
\mathscr{R}=\left\{\mathbf{x} \in R^{2}:|G \mathbf{x}| \leq 0.4358 \gamma\right\}
$$

is entirely contained in the region $X_{K_{0}}$. Hence a suitable value for $\Lambda$ is 0.4358 . Figure 3 shows the response of the controller with initial conditions $(1,0.3)$, a quantization $\|u\|_{x} \leq 0.0071$, and 


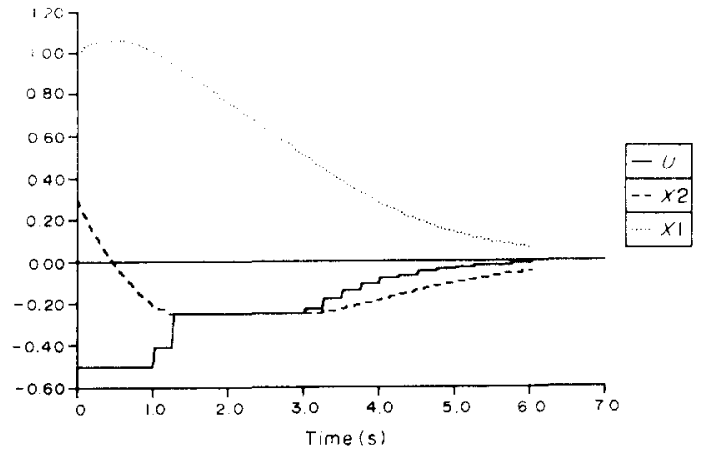

FIG. 3. Closed-loop system response for Example 2

computation time restricted to $0.25 \mathrm{~s}$. For this system the H.E.O.C. algorithm is in general faster than the On-Line Quadratic Programming employed in Sznaier and Damborg (1987), allowing for a more accurate solution (i.e. a solution that takes more terms into account). Note that the conditions of Theorem 4 that restrict the norm of the quantization to 0.0071 are overly conservative. Experimenting with this problem we have obtained convergence to the region $X_{K_{0}}$ with quantizations of up to 0.5 .

\section{A different heuristic}

The simple heuristic presented in the previous example works well when the system is not very far from the unconstrained region and the costs on the states and controls are comparable. However, this heuristic may badly underestimate the cost-to-go. When far from the unconstrained region, most of the cost for a quadratic regulator problem is associated with the states and the control cost is essentially constant and negligible. Under these conditions, the heuristic derived from the unconstrained linear quadratic problem gives a poor estimate since it assumes that the system is going to proceed to the origin with a large control effort that invariably exceeds the boundary. As a result, the system remains in the high cost region for a longer time than estimated by the heuristic. The poor estimate of the cost causes the algorithm to almost completely expand one level of the tree before proceeding to the next level, thereby rapidly running out of resources and resulting in poor performance. This difficulty can be solved by using a lower bound of the cost based upon the distance to the origin and an estimate of the number of steps necessary to reach it.

Consider the case when the control is restricted to the hypersphere $\Omega=\{\mathbf{u} \epsilon$ $\left.R^{m}:\|\mathbf{u}\|_{2} \leq r\right\}^{*}$ and let $\mathbf{x}_{k}$ be the present state of the system. It is clear that outside the region $C_{1}$

\footnotetext{
* This condition is not overly restrictive since we can always find a ball $B(0, r) \subseteq \Omega$
}

the control $\mathbf{u}$ that minimizes:

$$
\left\|x_{k+1}\right\|_{2}^{2}=\left(A \mathbf{x}_{k}+B \mathbf{u}_{k}\right)^{T}\left(A \mathbf{x}_{k}+B \mathbf{u}_{k}\right)
$$

is on the boundary of the set $\Omega$. Therefore:

$$
\begin{gathered}
\left\|\mathbf{x}_{k+1}\right\|_{2}^{2}=\mathbf{x}_{k}^{T} A^{T} A \mathbf{x}_{k}+2 \mathbf{x}_{k}^{T} A^{T} B \mathbf{u}_{k}+\mathbf{u}_{k}^{T} B^{T} B \mathbf{u}_{k} \\
\geq \mathbf{x}_{K}^{T} A^{T} A \mathbf{x}_{k}+2 \mathbf{x}_{K}^{T} A^{T} B \mathbf{u}_{k}+\left(\sigma_{\min }(B) r\right)^{2}
\end{gathered}
$$

where $\sigma_{\min }(B)$ indicates the minimum singular value of the matrix $B$. The value of $\mathbf{u}_{k}$ that minimizes (31) is given by:

$$
\mathbf{u}_{k}=\frac{-K \mathbf{x}_{k}}{\left\|K \mathbf{x}_{k}\right\|_{2}} r, \quad K=B^{T} A .
$$

Substituting (32) into (31) we get:

$$
\begin{array}{r}
\left\|\mathbf{x}_{k+1}\right\|_{2}^{2} \geq \sigma_{\min }(A)^{2}\left\|\mathbf{x}_{k}\right\|_{2}^{2}-2 \sigma_{\max }(K)\left\|\mathbf{x}_{k}\right\|_{2} \\
+\left(\sigma_{\min }(B) r\right)^{2}=\left[G\left(\left\|\mathbf{x}_{k}\right\|_{2}\right)\right]^{2}
\end{array}
$$

where:

$$
\begin{gathered}
\sigma_{\min }(A)=\text { minimum singular value of } A \\
\sigma_{\max }(K)=\text { maximum singular value of } K .
\end{gathered}
$$

Let:

$$
H\left(\left\|\mathbf{x}_{k}\right\|_{2}\right)=\left(\max \left\{0, G\left(\left\|\mathbf{x}_{k}\right\|_{2}\right)\right\}\right)^{2} .
$$

In the region:

$$
D=\left\{\mathbf{x} \in R^{n}:\|\mathbf{x}\|_{2} \geq \frac{\sigma_{\max }(K)}{\sigma_{\min }(A)}\right\}
$$

the operator $H$ is non-decreasing. Defining:

$$
L_{k}^{*}=0.5\left(\mathbf{x}_{k+1}^{T} Q \mathbf{x}_{k+1}+\mathbf{u}_{k}^{T} R \mathbf{u}_{k}\right) \geq 0.5 \mathbf{x}_{k+1}^{T} Q \mathbf{x}_{k+1}
$$

the cost can be expressed as:

$$
\begin{aligned}
J(\mathbf{x}, \mathbf{u}) & =\sum_{k=0}^{\infty} L_{k}^{*}+0.5\left(\mathbf{x}_{0}^{T} Q \mathbf{x}_{0}\right) \geq \sum_{k=0}^{\infty} 0.5 q\left\|\mathbf{x}_{k}\right\|_{2}^{2} \\
& \geq \sum_{k=0}^{N-1} 0.5 q\left\|\mathbf{x}_{k}\right\|_{2}^{2} \geq 0.5 q \sum_{k=0}^{N-1}\left[G^{k}\left(\left\|\mathbf{x}_{0}\right\|_{2}\right)\right]^{2} \\
& =0.5 q F\left(\left\|\mathbf{x}_{0}\right\|_{2}\right)=g_{2}(\mathbf{x})
\end{aligned}
$$

where:

$q=$ minimum eigenvalue of $Q$

$N=\min \left\{N_{1}=\right.$ minimum time to get to the origin, $N_{2}=$ instant where the system exits the region $D$.

It is clear that this approximation works best in the region far from the origin, where the term ${ }_{2} \mathbf{x}_{k+1}^{T} Q \mathbf{x}_{k+1}$ dominates $L_{k}$, while the heuristics $g_{1}(\mathbf{x})=\frac{1}{2} \mathbf{x}^{T} S \mathbf{x}$ works best close to the unconstrained region.

\section{Example 3}

Consider the design of a Quadratic Regulator for the spinning space station problem intro- 


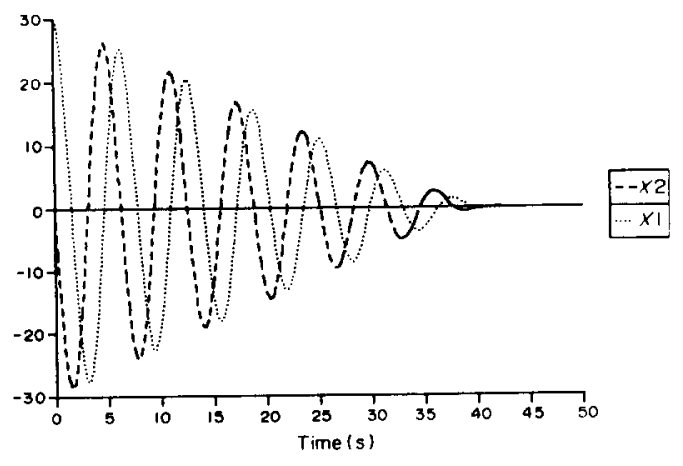

FIG. 4a. States for Example 3.

duced earlier. Let $Q=R=I$ and $T=2.5 \mathrm{~s}$. In this case it is clear that outside the region $C_{1}$ the term $\mathbf{x}^{T} \mathbf{x}$ dominates the cost (since $\mathbf{u}^{T} \mathbf{u}=1$ ). Hence, in that region we expect the heuristics introduced in the previous section to yield better results than $\frac{1}{2} \mathbf{x}^{T} S \mathbf{x}$. In this particular case we have:

$$
\begin{gathered}
\sigma_{\min }(A)=1, \quad \sigma_{\min }(B)=\sigma_{\max }(K)=\alpha, \quad q=1, \\
G\left(\left\|\mathbf{x}_{k}\right\|_{2}\right)=\left\|\mathbf{x}_{k}\right\|_{2}-\alpha \\
D=\left\{\mathbf{x} \in R^{n}:\|\mathbf{x}\|_{2} \geq \alpha\right\}=\bar{C}_{1},
\end{gathered}
$$

hence $N_{1}=N_{2}=N$ and

$$
\begin{aligned}
J(\mathbf{x}) \geq & 0.5 \sum_{k=0}^{N-1}\left(\left\|\mathbf{x}_{0}\right\|_{2}-k \alpha\right)^{2} \\
= & 0.5\left(N\left\|\mathbf{x}_{0}\right\|_{2}^{2}-N(N-1) \alpha\left\|\mathbf{x}_{0}\right\|\right. \\
& \left.+\frac{N(N-1)(2 N-1)}{6} \alpha^{2}\right) \\
= & g_{2}(\mathbf{x}) .
\end{aligned}
$$

Figure 4 shows the response of the controller to the initial condition $x^{T}=(30,0)$ using a combination of the heuristics $g_{1}$ and $g_{2}$. At a given state, we utilized the heuristics that yielded the highest estimate of the cost-to-go. As expected this combination provided a better performance than using the heuristic $g_{1}$ alone.

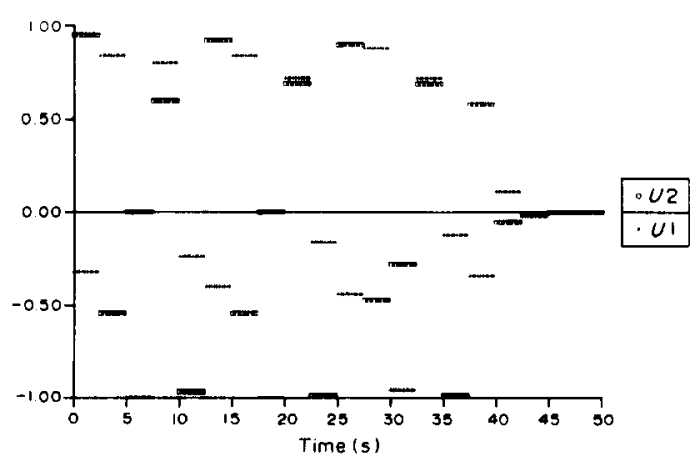

Fig. 4b. Controls for Example 3.

\section{CONCLUSIONS}

Most realistic control problems involve some types of constraints. However, to date there are no algorithms that allow dealing with constraints in a systematic way, except in some restricted cases. In the first part of the paper we developed a theoretical framework to analyze the stability properties of the closed-loop system resulting from the use of on-line optimization in the feedback loop. Our results show that under certain conditions the resulting closed-loop system is asymptotically stable in the region of interest and that the performance of the proposed controller approaches the performance of the true optimal controller for a modified problem, when the sampling interval is large.

In the second portion of the paper the results are specialized for the case where the on-line optimization is performed by casting the problem into a tree search form through the discretization of the control space. Subsequently, heuristic search techniques, based upon an under-estimate of the cost, are used to search the tree for minimum cost paths. This technique has the advantage of incorporating into the controller knowledge available about the solution, thus providing the potential for significant reductions in the computation time.

We believe that the suboptimal controller that we propose may provide significant advantages over the controllers available at the present time for the control of constrained systems. In particular we think that these controllers may be valuable for situations where the classical approaches of storing a family of extremal curves or solving a Hamilton-Jacobi equation in real time are not applicable. We expect that for these situations our approach will yield a systematic design procedure that incorporates optimality conditions and knowledge available about the system, as opposed to commonly used ad hoc techniques.

There are many open questions which remain to be resolved. At this point we are working in an estimation of the null controllability regions based upon the singular value decomposition of the matrix $A$ and the geometric properties of the sets $\Omega$ and $C_{1}^{u}$.

As we noted in the paper, even though the conditions presented that guaranteed asymptotic stability for a general algorithm are not very restrictive, the results of Lemma 1 and Theorem 4 , used in the implementation of Algorithm $\mathrm{H}$, proved to be overly restrictive in some cases. [Recall that equation (17) is the result of a "worst-case" type analysis.] As the norm of the partition gets smaller, the number of nodes of the tree that have to be checked increases and 
the problem may become intractable. This phenomenon, dubbed "the curse of dimensionality" by Bellman is common in dynamic programming approaches. Further, in equation (17) we did not take advantage of the full capabilities of Algorithm $\mathrm{H}$. Since for some problems it is known from theoretical considerations that the optimal controls lie on the boundary of the control set $\Omega$, this information should be incorporated into the controller and used to limit the search. This topic is the subject of current research and a future article is planned to report the results.

Finally, application of the proposed controller to performance indexes different from the ones presented in the paper and suitable heuristics should be investigated.

\section{REFERENCES}

Athans, M. and P. L. Falb (1966). Optimal Control. McGraw-Hill, New York.

Baker, D. R. (1987). Exact solution to some minimum time problems and their behavior near inequality state constraints. Proc. 26th IEEE Conf. Decis. Control, Los Angeles, CA, 9-11 December 1987, 1622-1626.

Dreyfus, S. E. (1964). Some types of optimal control of stochastic systems. J. SIAM Control A, 2, 120-134.

Fegley, K. A., S. Blum, J. O. Bergholm, A. J. Calise, J. E. Marowitz, G. Porcelli and L. P. Sinha (1971). Stochastic and deterministic design and control via linear and quadratic programming. IEEE Trans. Aut. Control, AC-16, 759-765.

Frankena, J. F. and R. Sivan (1979). A nonlinear optimal control law for linear systems. Int. J. Control, 30, 159-178.

Guez, A. (1986). Heuristically enhanced optimal control. Proc. 25th IEEE Conf. Decis. Control, Athens, Greece, 633-637.

Gutman, P. O. (1986). A linear programming regulator applied to hydroelectric reservoir level control. Automatica, 22, 533-541.

Gutman, P. O. and P. Hagander (1985). A new design of constrained controllers for linear systems. IEEE Trans. Aut. Control, AC-30, 22-33.

Mayne, D. Q. and E. Polak (1987). An exact penalty function algorithm for control problems with state and control constraints. IEEE Trans. Aut. Control, AC-32, $380-387$.

Sznaier, M. and M. J. Damborg (1987). Suboptimal control of linear systems with state and control inequality constraints. Proc. 26th IEEE Conf. Decis. Control. Los Angeles, CA, 9-11 December, 761-762.
Sznaier, M. and M. J. Damborg (1989). Control of constrained discrete time linear systems using quantized controls. Automatica, 25, 623-628.

Teren, F. (1977) Minimum-time acceleration of aircraft turbofan engines. Proc. 1977 Joint Aut. Control Conf. 22-24 June 1977, 1029-1037.

Van Til, R. P. and W. E. Schmittendorf, (1986) Constrained controllability of discrete-time systems. Int. $J$. Control, 43, 941-956.

Vassilaki, M., J. C. Kennet and G. Bitsoris (1988). Feedback control of discrete-time systems under state and control constraints. Int. J. Control, 47, 1727-1735.

Winston, P. H. (1984). Artificial Intelligence, 2nd edn., Chapter 4. Addison-Wesley, MA.

Zadeh, L. A. and B. H. Whalen (1962). On optimal control and linear programming. IRE Trans. Aut. Control, AC-7, $45-46$.

\section{APPENDIX}

\section{The tree search algorithm}

The algorithm utilized to expand and scan the tree is a modification of Algorithm $A^{*}$ presented by Winston (1984). The modification is required to assure that when there is not enough computation time available to completely expand the tree, the control utilized corresponds to the last completely explored level. The algorithm is as follows:

(1) Form a queue of partial paths, with the initial queue consisting of the zero-cost, zero-step path from the root node to nowhere. Set the level indicator to 0 .

(2) Until the queue is empty or the goal has been reached determine if the first path in the queue reaches the goal.

(2a) If the first path reaches the goal, do nothing.

(2b) If the first path does not reach the goal:

(2b1) Remove the first path from the queue. If the level of the tree corresponding to this path is not marked then mark the previous level as completed and store the control associated with this path as corresponding to the current level indicator. Update the level indicator.

(2b2) If possible, form new paths by extending the removed path one step with all permissible controls.

(2b3) Add the new paths that verify the state constraints to the queue. Set the level indicator of each path to the current level +1 .

(2b4) Sort the queue by the sum of the actual cost accumulated so far and a lower bound of the cost-to-go, with least-cost paths in front.

(3) If out of time use the control corresponding to the current level indicator -1 .

(4) If the goal has been reached announce success; otherwise announce failure. 